\title{
Synthesis of 2-(2-phenylethenyl) substituted 4,5-dihydrofurans by regioselective addition of 1,3-dicarbonyl compounds to dienes promoted by cerium(IV) ammonium nitrate
}

\author{
Mehmet Yilmaz* and Aslı Ustalar \\ Department of Chemistry, Faculty of Arts and Sciences, Kocaeli University, \\ 41380 Umuttepe, Kocaeli, Turkey \\ E-mail:mehmet.yilmaz@,kocaeli.edu.tr
}

DOI: http://dx.doi.org/10.3998/ark.5550190.p009.455

\begin{abstract}
Radical addition of 1,3-dicarbonyl compounds to conjugated dienes in the presence of cerium(IV) ammonium nitrate in THF produced 4,5-dihydrofurans in good to excellent yields. All radical additions occurred on the terminal double bond as regioselective. Two different dihydrofurans were obtained from the reaction of 1-phenyl-1,3-butanedione with 1-phenyl-1,3butadiene and 3-methyl-1-phenyl-1,3-butadiene. All compounds were characterised by IR, ${ }^{1} \mathrm{H}$, ${ }^{13} \mathrm{C}-\mathrm{NMR}$ and HRMS spectra.
\end{abstract}

Keywords: Cerium(IV) ammonium nitrate, oxidative addition, dihydrofuran, conjugated diene.

\section{Introduction}

It is well known that $\mathrm{Mn}(\mathrm{OAc})_{3}{ }^{1-14}$ and $\left(\mathrm{NH}_{4}\right)_{2} \mathrm{Ce}\left(\mathrm{NO}_{2}\right)_{6}(\mathrm{CAN}){ }^{15-24}$ are widely used as radical oxidants in the synthesis of poly functional organic compounds forming $\mathrm{C}-\mathrm{C}$ bond between basic compounds. These radical oxidants enable formation of dihydrofurans obtained from reaction of 1,3-dicarbonyls, 3-oxopropanenitriles, $\beta$-ketoesters and their derivatives with alkenes easily prepared by basic methods.

Our research group has focused the synthesis of dihydrofuran derivatives by the radical addition of various activated methyl ketones to unsaturated units such as alkenes, alkynes, dienes, and acrylamides. Recently, we have prepared 2,3-dihydro-4H-furo[3,2-c]chromen-4-ones and 2,3-dihydronaphtho[2,3-b]furan-4,9-diones by the cyclization of 4-hydroxycoumarin and 2hydroxy-1,4-naphthoquinone, respectively. ${ }^{25,26}$ Also, we carried out the reactions of fluorinated1,3-dicarbonyl compounds with dienes ${ }^{27}$ and conjugated alkenes, ${ }^{28-31}$ resulting in fluoroacetylated and fluoroalkylated 4,5-dihydrofurans. Moreover, we reported reactions of 1,3dicarbonyl compounds with alkynes ${ }^{32}$ and various substituted alkenes. ${ }^{33-36}$ Very recently, we have prepared 4,5-dihydrofuran-carbonitriles by the treatment of 3-oxopropanenitriles with 
alkenes, ${ }^{37-39}$ unsaturated amides ${ }^{40,41}$ and esters ${ }^{41}$ using $\mathrm{Mn}(\mathrm{OAc})_{3}$. In these reactions, $\mathrm{Mn}(\mathrm{OAc})_{3}$ was frequently used as radical oxidant in the presence of HOAc. However, recently we performed an optimization study on the radical addition of 3-oxopropanenitriles to alkenes using CAN in ethereal solvents, resulting CAN / THF system formed dihydrofurans in high yields on mild condition. ${ }^{42}$ In the present study, we applied the previous method to reactions of 1,3-dicarbonyl compounds with conjugated dienes promoted CAN / THF and obtained 2-(2phenylethenyl) substituted 4,5-dihydrofurans as regioselectively in excellent yields.

\section{Results and Discussion}

Conjugated dienes 1-phenyl-1,3-butadiene $2 \mathbf{a}^{43}$ and 3-methyl-1-phenyl-1,3-butadiene $\mathbf{2 b}{ }^{44}$ were synthesized from the reaction of methyl-triphenylphosphonium bromide and suitable carbonyl compounds in the presence of NaH/THF. 1,1-Diphenyl-1,3-butadiene 2c ${ }^{45}$ was prepared from water elimination of alcohol obtained from Grignard reaction of benzophenone and allylmagnesium bromide.

Radical addition of dimedone $\mathbf{1 a}$ and 1,3-cyclohexanedione 1b to 1-phenyl-1,3-butadiene 2a gave 2-(2-phenylethenyl) dihydrofuran $\mathbf{3 a}(85 \%)$ and $\mathbf{3 b}(80 \%)$ in excellent yields, respectively (Table 1). Also, treatment of 5-phenyl-1,3-cyclohexanedione 1c with 2a produced dihydrofuran 3c in $78 \%$ yield as diastereomeric mixture (determined by ${ }^{1} \mathrm{H}$ NMR spectrum, $\mathrm{dr}=50: 50$ ) of. Moreover, the reaction of 2,4-pentanedione 1d and ethyl 3-oxobutanoate 1e with 2 a occur 3d $(65 \%)$ and $3 \mathbf{e}(63 \%)$ in good yields, respectively. However, two different cyclic products $3 \mathbf{f}$ and $\mathbf{3 g}$ were obtained from the reaction of 1-phenyl-1,3-butanedione $\mathbf{1 f}$ and $\mathbf{2 a}$ since $\mathbf{1 f}$ have two different enol forms. These products $\mathbf{3 f}$ and $\mathbf{3 g}$ were differentiated by the chemical shift of the carbonyl carbons in their ${ }^{13} \mathrm{C}$ NMR spectra which show 195 ppm for $\mathbf{3 f}$ and 193.4 ppm for 3g. Also, The ${ }^{1} \mathrm{H}$ NMR spectrum of compound $\mathbf{3 g}$ show that protons $\mathrm{H} 4$ resonate with methyl group on the $\mathrm{C}-2$ carbon $\left({ }^{5} \mathrm{~J} 1.6 \mathrm{~Hz}\right)$ as long range coupling, but same coupling is not observed in the spectrum of $\mathbf{3 f}$.

Treatments of 1a and 1d with 1,1-diphenyl-1,3-butadiene $\mathbf{2 c}$ gave dihydrofuran $\mathbf{3 h}$ (91\%) (obtained in $78 \%$ yield by $\left.\mathrm{Mn}(\mathrm{OAc})_{3}\right)^{14}$ and $\mathbf{3 i}(84 \%)$ in excellent yields, respectively (Entries, 7 and 8). Upon comparing the addition reactions of both compounds $\mathbf{2 a}$ and $\mathbf{2 c}$, it is observed that diene 2c produced dihydrofuran in higher yield. This occurrence can be explained with the stability of intermediate radical formed in 1,3-dicarbonyl. Since 2 c has two phenyl groups, radical group of it is more stable compared to that of $\mathbf{2 a}$ 's.

It was reported in the literature that radical reaction of dimedone 1a with 1-phenyl-3-methyl1,3-butadiene $\mathbf{2 b}$ produced $\mathbf{3 j}$ by using $\mathrm{PbI}(\mathrm{OAc})_{2}(69 \%){ }^{46}$ and $\mathrm{CAN} / \mathrm{MeOH}(40 \%){ }^{47}$ as radical oxidants. But in this work, dihydrofuran $\mathbf{3 j}$ was obtained in $92 \%$ yield using CAN/THF system. Also, 2-(2-phenylethenyl) substituted dihydrofuran $3 \mathbf{k}(88 \%)$ and diastereomeric mixture $(\mathrm{dr}=$ 50:50) of 31 (82\%) were obtained in excellent yields (entries 4 and 5). Similarly, while it was reported that synthesis of compounds $\mathbf{3 m}$ and $\mathbf{3 n}$ through $\mathrm{CAN} / \mathrm{MeOH}$ in $45 \%$ and $40 \%$ yields, 
respectively, ${ }^{47}$ we obtained these compounds in very good yields ( $75 \%$ and $71 \%$, respectively) by using THF as solvent.

The reaction of $\mathbf{1 f}$ with $\mathbf{2 b}$ gave two different cyclic products $\mathbf{3 o}$ and $\mathbf{3 p}$ in moderate yields. These compounds were differentiated by the chemical shift of carbonyl groups in their ${ }^{13} \mathrm{C} N M R$ spectra as mentioned above.

Table 1. Radical addition of 1,3-dicarbonyl compounds (1a-f) to conjugated dienes (2a-c)<smiles>[R]C(=O)CC([R])=O</smiles>

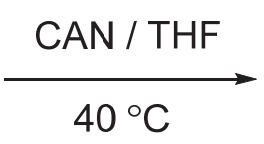<smiles>[R]C(=O)C1=C([R])OC([R])(/C=C(/[R])c2ccccc2)C1</smiles>

\begin{tabular}{|c|c|c|c|c|c|c|}
\hline Entry & $\mathrm{R}^{1}$ & & $\mathrm{R}^{3}$ & $\mathrm{R}^{4}$ & & $\begin{array}{c}\text { Products and } \\
\text { yields }{ }^{\mathrm{a}}(\%)\end{array}$ \\
\hline 1 & $-\mathrm{CH}_{2} \mathrm{C}\left(\mathrm{CH}_{3}\right)_{2} \mathrm{CH}_{2}-$ & $1 \mathbf{a}$ & $\mathrm{H}$ & $\mathrm{H}$ & $\mathbf{2 a}$ & $\mathbf{3 a}(85)$ \\
\hline 2 & $-\mathrm{CH}_{2} \mathrm{CH}_{2} \mathrm{CH}_{2-}$ & 1b & $\mathrm{H}$ & $\mathrm{H}$ & $\mathbf{2 a}$ & $\mathbf{3 b}(80)$ \\
\hline 3 & $-\mathrm{CH}_{2} \mathrm{CHPhCH}_{2-}$ & 1c & $\mathrm{H}$ & $\mathrm{H}$ & $\mathbf{2 a}$ & $\mathbf{3 c}(78)$ \\
\hline 4 & $-\mathrm{CH}_{3}$ & 1d & $\mathrm{H}$ & $\mathrm{H}$ & $\mathbf{2 a}$ & 3d (65) \\
\hline 5 & $-\mathrm{OCH}_{2} \mathrm{CH}_{3}$ & $1 \mathrm{e}$ & $\mathrm{H}$ & $\mathrm{H}$ & $\mathbf{2 a}$ & $3 e(63)$ \\
\hline 6 & $-\mathrm{CH}_{3}$ & 1f & $\mathrm{H}$ & $\mathrm{H}$ & $\mathbf{2 a}$ & $\begin{array}{l}\mathbf{3 f}(27)^{\mathrm{b}} \\
\mathbf{3 g}(39)^{\mathrm{c}}\end{array}$ \\
\hline 7 & $-\mathrm{CH}_{2} \mathrm{C}\left(\mathrm{CH}_{3}\right)_{2} \mathrm{CH}_{2}-$ & $1 \mathbf{a}$ & $\mathrm{H}$ & $\mathrm{Ph}$ & $2 c$ & 3h (91) \\
\hline 8 & $\mathrm{CH}_{3}$ & 1d & $\mathrm{H}$ & $\mathrm{Ph}$ & $2 \mathrm{c}$ & $3 \mathbf{i}(84)$ \\
\hline 9 & $-\mathrm{CH}_{2} \mathrm{C}\left(\mathrm{CH}_{3}\right)_{2} \mathrm{CH}_{2}-$ & $1 \mathbf{a}$ & $\mathrm{CH}_{3}$ & $\mathrm{H}$ & $\mathbf{2 b}$ & 3j (92) \\
\hline 10 & $-\mathrm{CH}_{2} \mathrm{CH}_{2} \mathrm{CH}_{2}-$ & $1 \mathbf{b}$ & $\mathrm{CH}_{3}$ & $\mathrm{H}$ & $2 \mathbf{b}$ & 3k (88) \\
\hline 11 & $-\mathrm{CH}_{2} \mathrm{CHPhCH}_{2-}$ & 1c & $\mathrm{CH}_{3}$ & $\mathrm{H}$ & $2 \mathbf{b}$ & $3 \mathbf{I}(82)$ \\
\hline 12 & $-\mathrm{CH}_{3}$ & 1d & $\mathrm{CH}_{3}$ & $\mathrm{H}$ & $2 \mathbf{b}$ & $3 \mathbf{m}(75)$ \\
\hline 13 & $-\mathrm{OCH}_{2} \mathrm{CH}_{3}$ & 1e & $\mathrm{CH}_{3}$ & $\mathrm{H}$ & $2 b$ & $3 n(71)$ \\
\hline 14 & $-\mathrm{CH}_{3}$ & 1f & $\mathrm{CH}_{3}$ & $\mathrm{H}$ & $2 \mathbf{b}$ & $\begin{array}{l}3 \mathbf{o}(40)^{\mathrm{b}} \\
\mathbf{3 p}(38)^{\mathrm{c}}\end{array}$ \\
\hline
\end{tabular}

${ }^{\mathrm{a}}$ Yields of isolated products based on the dienes. ${ }^{\mathrm{b}}$ For the compounds $\mathbf{3 f}$ and $\mathbf{3 o}, \mathrm{R}^{1}=\mathrm{CH}_{3}, \mathrm{R}^{2}=$ $\mathrm{Ph}$ c: For the compounds $\mathbf{3 g}$ and $\mathbf{3 p}, \mathrm{R}^{1}=\mathrm{Ph}, \mathrm{R}^{2}=\mathrm{CH}_{3}$. 
The mechanism proposed for the radical addition of conjugated dienes 2a-c with 1,3dicarbonyls 1a-f is presented in Scheme 1. According to this mechanism, while $\mathrm{Ce}^{+4}$ is reduced to $\mathrm{Ce}^{+3}$ a radical cation $\mathbf{B}$ is formed. ${ }^{48}$ Then, addition of the radical to the terminal double bond of the diene forms an allylic radical intermediate $\mathbf{C}$. Radical $\mathbf{C}$ is oxidized to the carbocation $\mathbf{D}$ by CAN, followed by cyclization of $\mathbf{D}$ to give 5-(2-phenylvinyl)-4,5-dihydrofuran $\mathbf{E}$. Intermediates $\mathbf{F}$ and $\mathbf{H}$ which is the another enol form of $\mathbf{F}$ were obtained from the radical addition of 1-phenyl-1,3-butanedione 1f to diene $\mathbf{2 b}$. Intramolecular cyclization of these intermediates gave dihydrofurans $\mathbf{G}$ and $\mathbf{I}$, respectively. All radical additions to the dienes occurred on the terminal double bond of the dienes as regioselectively, other adduct products were not observed.

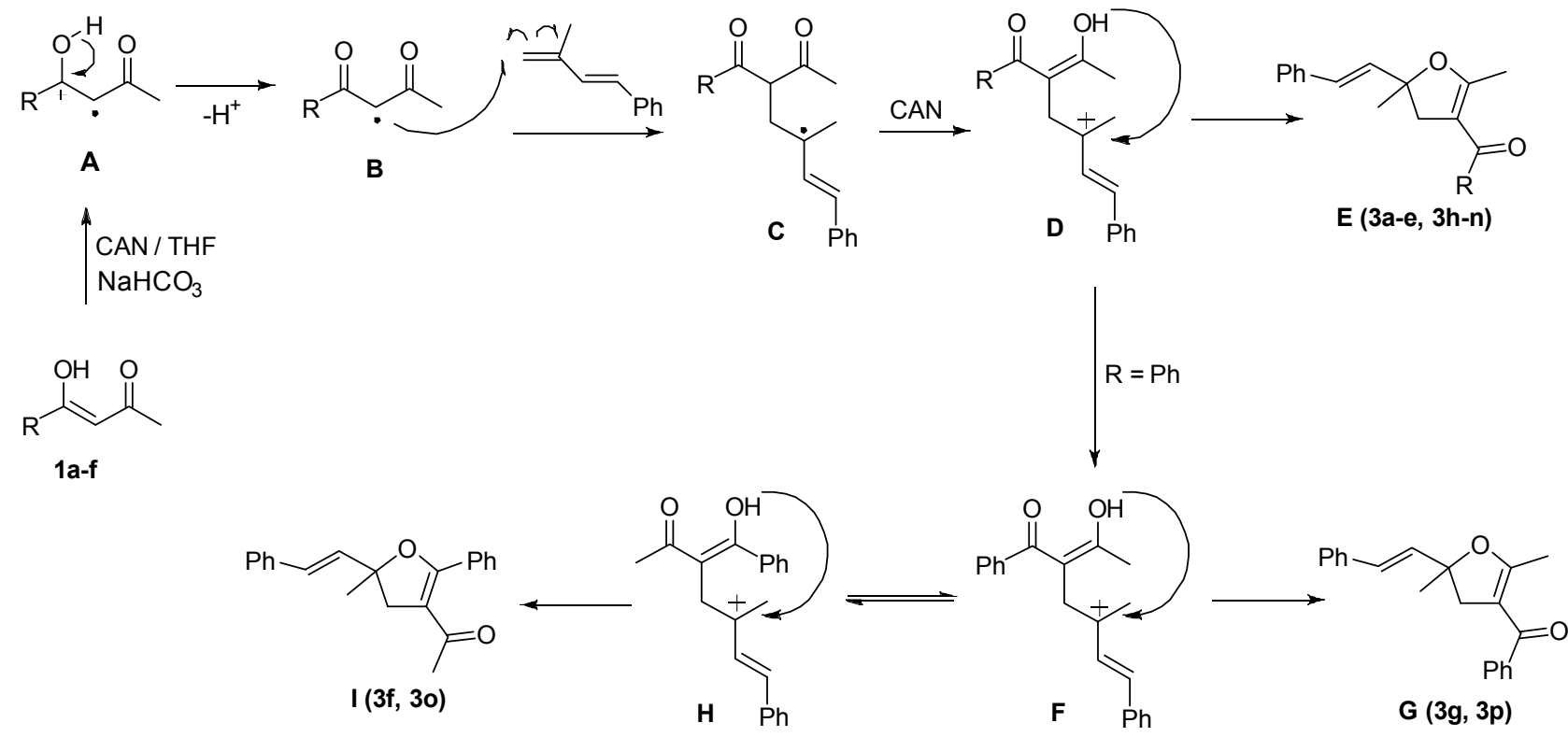

Scheme 1. Mechanism proposed for the formation of dihydrofurans.

\section{Conclusions}

Cerium(IV) amonium nitrate promoted radical addition of 1,3-dicarbonyl compounds to 1,3butadiene derivatives was investigated, resulting in formation of various 2-phenylvinyl-4,5dihydrofurans. Previous methodology that was optimized using alkenes was applied here to radical addition of 1,3-dicarbonyls to dienes. Upon doing a literature review and comparing the results, it is deduced that, in the radical addition of dienes to 1,3-dicarbonyls promoted CAN/THF system can be used in a highly effective way. 


\section{Experimental Section}

General. Melting points were determined on an electrothermal capillary melting point apparatus. IR spectra (ATR) were obtained with a Bruker Tensor-27 400-4000 $\mathrm{cm}^{-1}$ range with $2 \mathrm{~cm}^{-1}$ resolution. ${ }^{1} \mathrm{H}$ NMR, ${ }^{13} \mathrm{C}$ NMR, spectra were recorded on a Bruker Avance DPX-400 MHz and Varian Oxford NMR300 High performance Digital FT-NMR spectrophotometers. High Resolution Mass Time-of-Flight (TOF) was measured on an Agilent 1200/6210 LC/MS spectrophotometer. The mass spectra were measured on a Waters-2695-Alliance-Micromass-ZQ instrument in $\mathrm{m} / \mathrm{z}$ (rel.\%). Elemental analyses were performed on a VarioEL III CHNS instrument. $\left[\mathrm{Mn}(\mathrm{OAc})_{3}\right] \cdot 2 \mathrm{H}_{2} \mathrm{O}$ was prepared by electrochemically method according to the literature ${ }^{4}$. Thin layer chromatography (TLC) was performed on Merck aluminum-packed silica gel plates. Purification of the products was performed by column chromatography on silica gel (Merck silica gel 60, 40-63 mm).

General procedure for the synthesis of 4,5-dihydrofurans (3a-p). To a soln. of 1,3dicarbonyls $(1 \mathrm{mmol})$ and diene $(1.5 \mathrm{mmol})$ in THF $(10 \mathrm{~mL})$ under $\mathrm{N}_{2}$ in an oil bath, a mixture of $\mathrm{CAN}(2.5 \mathrm{mmol})$ and $\mathrm{NaHCO}_{3}(2.5 \mathrm{mmol})$ was added at $30^{\circ} \mathrm{C}$. Then, the temp. was slowly increased to $40^{\circ}$. The reaction was completed when the orange colour of CAN had disappeared (10 min) or when the diene spot on TLC had completely vanished. $\mathrm{H}_{2} \mathrm{O}$ was added to the soln., and the mixture was extracted with $\mathrm{CHCl}_{3}(3 \times 20 \mathrm{~mL})$. The combined organic phase was dried $\left(\mathrm{Na}_{2} \mathrm{SO}_{4}\right)$ and concentrated and the crude product purified by column chromatography on silica gel $(230-400 \mathrm{mesh})$ or preparative TLC (20x20 cm plates, $2 \mathrm{~mm}$ thickness, n-hexane/EtOAc ( 5 $: 1)$.

6,6-Dimethyl-2-[(E)-2-phenylvinyl]-3,5,6,7-tetrahydro-1-benzofuran-4(2H)-one (3a). Light yellow oil, yield 85\%, $228 \mathrm{mg}$, IR (ATR, $\left.\mathrm{cm}^{-1}\right)$ : 3058, 2961, $1695(\mathrm{C}=\mathrm{O}), 1625(\mathrm{C}=\mathrm{C}), 1020$, 756, 696. ${ }^{1} \mathrm{H}$ NMR $\left(400 \mathrm{MHz}, \mathrm{CDCl}_{3}\right), \delta_{\mathrm{H}} 1.09\left(6 \mathrm{H}, \mathrm{s}, 2 \mathrm{xCH}_{3}\right), 2.22(2 \mathrm{H}, \mathrm{s}, \mathrm{H} 5), 2.30(2 \mathrm{H}, \mathrm{s}$, H7), $2.70(1 \mathrm{H}, \mathrm{dd}, J 14.4$ and $7.6 \mathrm{~Hz}, \mathrm{H} 3), 3.07(1 \mathrm{H}, \mathrm{dd}, J 14.4$ and $10.4 \mathrm{~Hz}, \mathrm{H} 3), 5.37(1 \mathrm{H}, \mathrm{dt}, J$ 10.4 and $7.6 \mathrm{~Hz}, \mathrm{H} 2), 6.22\left(1 \mathrm{H}, \mathrm{dd}, J 16.0\right.$ and $\left.7.6 \mathrm{~Hz}, \mathrm{H}_{\text {olef. }}\right), 6.62\left(1 \mathrm{H}, \mathrm{d}, J 16.0 \mathrm{~Hz}, \mathrm{H}_{\text {olef. }}\right), 7.25$ $(1 \mathrm{H}, \mathrm{t}, J 7.2 \mathrm{~Hz}), 7.30(2 \mathrm{H}, \mathrm{t}, J 7.2 \mathrm{~Hz}), 7.36(2 \mathrm{H}, \mathrm{d}, J 7.6 \mathrm{~Hz}) .{ }^{13} \mathrm{C} \mathrm{NMR}\left(100 \mathrm{MHz}, \mathrm{CDCl}_{3}\right), \delta_{\mathrm{C}}$ $28.9\left(\mathrm{CH}_{3}\right), 28.95\left(\mathrm{CH}_{3}\right), 32.3,34.3,38.0,51.2(\mathrm{C} 3), 86.4(\mathrm{C} 2), 111.7(\mathrm{C} 3 \mathrm{a}), 127.0,127.4$ $(2 \times C H), 128.5(2 \times C H), 128.9,133.2,136.0,176.2(\mathrm{C7a}), 194.5(\mathrm{C}=\mathrm{O}) . \mathrm{m} / z\left(\mathrm{ESI}^{+}\right)=269\left(\mathrm{MH}^{+}\right.$, 100\%). HRMS $\left(\mathrm{ESI}^{+}\right): m / z(\mathrm{M}+\mathrm{H})^{+} \mathrm{C}_{18} \mathrm{H}_{20} \mathrm{O}_{2}: 269.15361$ found: 269.15461 . Anal. Calcd for $\mathrm{C}_{18} \mathrm{H}_{20} \mathrm{O}_{2}$ (268.35): $\mathrm{C}, 80.56 ; \mathrm{H}, 7.51 \%$. Found: $\mathrm{C}, 80.42 ; \mathrm{H}, 7.73 \%$.

2-[(E)-2-Phenylvinyl]-3,5,6,7-tetrahydro-1-benzofuran-4(2H)-one (3b). Yellow solid, yield

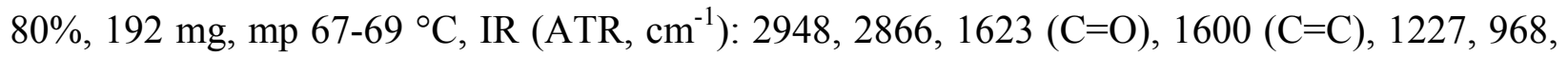
756, 691. ${ }^{1} \mathrm{H}$ NMR $\left(400 \mathrm{MHz}, \mathrm{CDCl}_{3}\right), \delta_{\mathrm{H}} 2.05(2 \mathrm{H}, \mathrm{f}, J 6.4 \mathrm{~Hz}, \mathrm{H} 6), 2.36(2 \mathrm{H}, \mathrm{t}, J 3.2 \mathrm{~Hz}, \mathrm{H} 5)$, $2.46\left(2 \mathrm{H}, \mathrm{tt}, J 6.4\right.$ and $\left.{ }^{5} J_{\mathrm{H} 7-\mathrm{H} 3}=1.6 \mathrm{~Hz}, \mathrm{H} 7\right), 2.7\left(1 \mathrm{H}, \mathrm{ddd}, J 14.4,8.0\right.$ and $\left.{ }^{5} J_{\mathrm{H} 3-\mathrm{H} 7}=1.6 \mathrm{~Hz}, \mathrm{H} 3\right)$, $3.1\left(1 \mathrm{H}\right.$, ddt, $J 14.4,10.4$ and $\left.{ }^{5} J_{\mathrm{H} 3}{ }^{\prime}-\mathrm{H} 7=1.6 \mathrm{~Hz}, \mathrm{H} 3\right), 5.30(1 \mathrm{H}$, ddd, $J 10.47 .6$ and $7.2 \mathrm{~Hz}, \mathrm{H} 2)$, $6.20\left(1 \mathrm{H}, \mathrm{dd}, J 16.0\right.$ and $\left.7.2 \mathrm{~Hz}, \mathrm{H}_{\text {olef. }}\right), 6.60\left(1 \mathrm{H}, \mathrm{d}, J 16.0 \mathrm{~Hz}, \mathrm{H}_{\text {olef. }}\right), 7.20(1 \mathrm{H}, \mathrm{tt}, J 7.6$ and 2.4 $\mathrm{Hz}), 7.30(2 \mathrm{H}, \mathrm{t}, J 7.6 \mathrm{~Hz}), 7.40(2 \mathrm{H}, \mathrm{d}, J 8.4 \mathrm{~Hz}) .{ }^{13} \mathrm{C} \mathrm{NMR}\left(100 \mathrm{MHz}, \mathrm{CDCl}_{3}\right), \delta_{\mathrm{C}} 21.9\left(\mathrm{CH}_{2}\right)$, 
$24.2\left(\mathrm{CH}_{2}\right), 32.5,36.7(\mathrm{C} 3), 86.2(\mathrm{C} 2), 113.3(\mathrm{C} 3 \mathrm{a}), 127.0,127.4(2 \mathrm{xCH}), 128.5(2 \times \mathrm{CH}), 128.9$, 133.3, 136.0, $177.3(\mathrm{C} 7 \mathrm{a}), 195.6(\mathrm{C}=\mathrm{O}) . \mathrm{m} / z\left(\mathrm{ESI}^{+}\right)=241\left(\mathrm{MH}^{+}, 100 \%\right)$. HRMS $\left(\mathrm{ESI}^{+}\right): \mathrm{m} / \mathrm{z}$ $(\mathrm{M}+\mathrm{H})^{+} \mathrm{C}_{16} \mathrm{H}_{16} \mathrm{O}_{2}: 241.12231$ found: 241.12334. Anal. Calcd for $\mathrm{C}_{16} \mathrm{H}_{16} \mathrm{O}_{2}$ (240.29): C, 79.97; H, $6.71 \%$. Found: $\mathrm{C}, 80.35 ; \mathrm{H}, 6.52 \%$.

6-Phenyl-2-[(E)-2-phenylvinyl]-3,5,6,7-tetrahydro-1-benzofuran-4(2H)-one (3c). Pale yellow solid, yield 78\%, $246 \mathrm{mg}$, mp 64-66 ${ }^{\circ} \mathrm{C}$, IR (ATR, $\left.\mathrm{cm}^{-1}\right)$ : 3029, 2954, 2937, $1623(\mathrm{C}=\mathrm{O}), 1600$ $(\mathrm{C}=\mathrm{C}), 1203,748,688 .{ }^{1} \mathrm{H}$ NMR $\left(300 \mathrm{MHz}, \mathrm{CDCl}_{3}\right), \delta_{\mathrm{H}}$ 2.56-2.70 (5H, m, H5, H6, H7), 3.07 $(1 \mathrm{H}, \mathrm{m}, \mathrm{H} 3), 3.38(1 \mathrm{H}, \mathrm{m}, \mathrm{H} 3), 5.35(1 \mathrm{H}, \mathrm{m}, \mathrm{H} 2), 6.20\left(1 \mathrm{H}, \mathrm{dd}, J 15.9\right.$ and $\left.7.5 \mathrm{~Hz}, \mathrm{H}_{\text {olef. }}\right), 6.6$ $\left(1 \mathrm{H}, \mathrm{d}, J 15.9 \mathrm{~Hz}, \mathrm{H}_{\text {olef. }}\right), 7.16-7.34(10 \mathrm{H}, \mathrm{m}) .{ }^{13} \mathrm{C} \mathrm{NMR}\left(75 \mathrm{MHz}, \mathrm{CDCl}_{3}\right), \delta_{\mathrm{C}} 31.8\left(\mathrm{CH}_{2}\right), 32.2$ $\left(\mathrm{CH}_{2}\right), 40.5,44.0(\mathrm{C} 3), 86.6(\mathrm{C} 2), 113.1(\mathrm{C} 3 \mathrm{a}), 127.0,127.05,127.1(2 \mathrm{xCH}), 127.4(2 \mathrm{xCH})$, $128.6(2 \times C H), 128.9,129.0,133.3,135.9,142.8,176.6(\mathrm{C} 7 \mathrm{a}), 194.2(\mathrm{C}=\mathrm{O}) . \mathrm{m} / z\left(\mathrm{ESI}^{+}\right)=317$ $\left(\mathrm{MH}^{+}, 100 \%\right)$. HRMS (ESI $\left.{ }^{+}\right): m / z(\mathrm{M}+\mathrm{H})^{+} \mathrm{C}_{22} \mathrm{H}_{20} \mathrm{O}_{2}: 317.15361$ found: 317.15315 . Anal. Calcd for $\mathrm{C}_{22} \mathrm{H}_{20} \mathrm{O}_{2}$ (316.39): C, 83.51; H, 6.37\%. Found: C, 83.85; H, 6.30\%.

1-\{2-Methyl-5-[(E)-2-phenylvinyl]-4,5-dihydrofuran-3-yl\}ethanone (3d). Yellow oil, yield 65\%, $148 \mathrm{mg}$, IR (ATR, cm ${ }^{-1}$ ): 3027, 2925, 1698 (C=O), 1592 (C=C), 1221, 929, 749, 693. ${ }^{1} \mathrm{H}$ NMR $\left(300 \mathrm{MHz}, \mathrm{CDCl}_{3}\right), \delta_{\mathrm{H}} 2.21\left(3 \mathrm{H}, \mathrm{s}, \mathrm{CH}_{3}\right), 2.25\left(3 \mathrm{H}, \mathrm{t}, J 1.5 \mathrm{~Hz}, \mathrm{CH}_{3}\right), 2.81(1 \mathrm{H}, \mathrm{ddq}, J$ 14.1, 8.1 and $1.5 \mathrm{~Hz}, \mathrm{H} 4), 3.17$ (1H, ddq, $J 14.1,10.5$ and $1.5 \mathrm{~Hz}, \mathrm{H} 4), 5.20$ (1H, ddd, $J 10.2,8.1$ and 7.2 Hz, H5), $6.23\left(1 \mathrm{H}, \mathrm{dd}, J 15.9\right.$ and $\left.7.5 \mathrm{~Hz}, \mathrm{H}_{\text {olef. }}\right), 6.60\left(1 \mathrm{H}, \mathrm{d}, J 15.9 \mathrm{~Hz}, \mathrm{H}_{\text {olef. }}\right), 7.25(1 \mathrm{H}$, t, $J 8.4 \mathrm{~Hz}), 7.31(2 \mathrm{H}, \mathrm{t}, J 6.9 \mathrm{~Hz}), 7.4(2 \mathrm{H}, \mathrm{d}, J 8.4 \mathrm{~Hz}) .{ }^{13} \mathrm{C} \mathrm{NMR}\left(75 \mathrm{MHz}, \mathrm{CDCl}_{3}\right), \delta_{\mathrm{C}} 15.3$ $\left(\mathrm{CH}_{3}\right), 29.7\left(\mathrm{CH}_{3}\right), 36.9(\mathrm{C} 4), 83.0(\mathrm{C} 5), 112.3(\mathrm{C} 3), 126.9,127.8(2 \times \mathrm{CH}), 128.5(2 \mathrm{xCH}), 128.9$, 132.8, 136.1, $167.8(\mathrm{C} 2), 194.8(\mathrm{C}=\mathrm{O}) . \mathrm{m} / \mathrm{z}\left(\mathrm{ESI}^{+}\right)=229\left(\mathrm{MH}^{+}, 100 \%\right)$. HRMS $\left(\mathrm{ESI}^{+}\right): \mathrm{m} / \mathrm{z}$ $(\mathrm{M}+\mathrm{H})^{+} \mathrm{C}_{15} \mathrm{H}_{16} \mathrm{O}_{2}: 229.12231$ found: 229.12284. Anal. Calcd for $\mathrm{C}_{15} \mathrm{H}_{16} \mathrm{O}_{2}$ (228.28): C, 78.92; $\mathrm{H}$, 7.06\%. Found: C, 79.05; H, 7.18\%.

Ethyl 2-methyl-5-[(E)-2-phenylvinyl]-4,5-dihydrofuran-3-carboxylate(3e). Pale yellow oil, yield 63\%, $163 \mathrm{mg}$, IR (ATR, $\left.\mathrm{cm}^{-1}\right): 2962,1692(\mathrm{C}=\mathrm{O}), 1644(\mathrm{C}=\mathrm{C}), 1078,786,692 .{ }^{1} \mathrm{H}$ NMR $\left(300 \mathrm{MHz}, \mathrm{CDCl}_{3}\right), \delta_{\mathrm{H}} 1.27\left(3 \mathrm{H}, \mathrm{t}, J 9.2 \mathrm{~Hz},-\mathrm{OCH}_{2} \mathrm{CH}_{3}\right), 2.20\left(3 \mathrm{H}, \mathrm{t}, J 1.5 \mathrm{~Hz}, \mathrm{CH}_{3}\right), 2.75(1 \mathrm{H}$, ddq, $J 14.1,8.1$ and $1.5 \mathrm{~Hz}, \mathrm{H} 4), 3.12(1 \mathrm{H}$, ddq, $J 14.4,10.2$ and $1.5 \mathrm{~Hz}, \mathrm{H} 4), 4.2(2 \mathrm{H}, \mathrm{q}, J 7.2$ $\left.\mathrm{Hz},-\mathrm{OCH}_{2} \mathrm{CH}_{3}\right), 5.20(1 \mathrm{H}$, ddd, $J 10.5,7.8$ and $6.9 \mathrm{~Hz}, \mathrm{H}$ ), $6.25(1 \mathrm{H}, \mathrm{dd}, J 15.9$ and $7.2 \mathrm{~Hz}$, $\left.\mathrm{H}_{\text {olef. }}\right), 6.60\left(1 \mathrm{H}, \mathrm{d}, J 15.9 \mathrm{~Hz}, \mathrm{H}_{\text {olef. }}\right), 7.25(1 \mathrm{H}, \mathrm{t}, J 6.6 \mathrm{~Hz}), 7.31(2 \mathrm{H}, \mathrm{t}, J 6.6 \mathrm{~Hz}), 7.4(2 \mathrm{H}, \mathrm{d}, J$ $6.9 \mathrm{~Hz}) .{ }^{13} \mathrm{C}$ NMR $\left(75 \mathrm{MHz}, \mathrm{CDCl}_{3}\right), \delta_{\mathrm{C}} 14.4\left(\mathrm{CH}_{3}\right), 14.7\left(\mathrm{CH}_{3}\right), 36.2(\mathrm{C} 4), 59.7,82.8(\mathrm{C} 5)$, $102.0(\mathrm{C} 3), 126.9,128.1(2 \mathrm{xCH}), 128.3,128.8(2 \mathrm{xCH}), 132.5,136.3,166.3(\mathrm{C} 2), 167.8(\mathrm{C}=\mathrm{O})$. $m / z\left(\mathrm{ESI}^{+}\right)=259\left(\mathrm{MH}^{+}, 100 \%\right)$. HRMS $\left(\mathrm{ESI}^{+}\right): m / z(\mathrm{M}+\mathrm{H})^{+} \mathrm{C}_{16} \mathrm{H}_{18} \mathrm{O}_{3}: 259.13287$ found: 259. 13231. Anal. Calcd for $\mathrm{C}_{16} \mathrm{H}_{18} \mathrm{O}_{3}$ (258.31): C, 74.39; H, 7.02\%. Found: C, 74.55; H, 6.75\%.

1-\{2-Phenyl-5-[(E)-2-phenylvinyl]-4,5-dihydrofuran-3-yl\}ethanone (3f). Yellow oil, yield 27\%, $78 \mathrm{mg}$, IR (ATR, $\left.\mathrm{cm}^{-1}\right): 2925,1717(\mathrm{C}=\mathrm{O}), 1677(\mathrm{C}=\mathrm{C}), 1595,750,693 .{ }^{1} \mathrm{H}$ NMR $\left(300 \mathrm{MHz}, \mathrm{CDCl}_{3}\right), \delta_{\mathrm{H}} 1.94\left(3 \mathrm{H}, \mathrm{s}, \mathrm{CH}_{3}\right), 3.0(1 \mathrm{H}, \mathrm{dd}, J 15.0$ and $8.4 \mathrm{~Hz}, \mathrm{H} 4), 3.32(1 \mathrm{H}, \mathrm{dd}, J$ 15.0 and $10.2 \mathrm{~Hz}, \mathrm{H} 4), 5.30(1 \mathrm{H}$, ddd, $J 10.0,8.4$ and $7.2 \mathrm{~Hz}, \mathrm{H} 5), 6.30(1 \mathrm{H}, \mathrm{dd}, J 15.9$ and 7.2 $\left.\mathrm{Hz}, \mathrm{H}_{\text {olef. }}\right), 6.65\left(1 \mathrm{H}, \mathrm{d}, J 15.9 \mathrm{~Hz}, \mathrm{H}_{\text {olef. }}\right), 7.22-7.30(3 \mathrm{H}, \mathrm{m}), 7.36-7.42(5 \mathrm{H}, \mathrm{m}), 7.30(2 \mathrm{H}, \mathrm{dd}, J$ 7.8 and $1.5 \mathrm{~Hz}) .{ }^{13} \mathrm{C}$ NMR $\left(75 \mathrm{MHz}, \mathrm{CDCl}_{3}\right), \delta_{\mathrm{C}} 29.1\left(\mathrm{CH}_{3}\right), 37.6(\mathrm{C} 4), 83.2(\mathrm{C} 5), 114.9(\mathrm{C} 3)$, 127.0, 127.7, $128.0(2 \mathrm{xCH}), 128.4(2 \mathrm{xCH}), 128.6(2 \mathrm{xCH}), 128.9(2 \mathrm{xCH}), 129.5,130.9,133.1$, 
136.2, $168.7(\mathrm{C} 2), 195.0(\mathrm{C}=\mathrm{O}) \cdot m / z\left(\mathrm{ESI}^{+}\right)=291\left(\mathrm{MH}^{+}, 100 \%\right) . \mathrm{HRMS}\left(\mathrm{ESI}^{+}\right): m / z(\mathrm{M}+\mathrm{H})^{+}$ $\mathrm{C}_{20} \mathrm{H}_{19} \mathrm{O}_{2}$ : 291.13796 found: 291.14053. Anal. Calcd for $\mathrm{C}_{20} \mathrm{H}_{18} \mathrm{O}_{2}$ (290.35): C, 82.73; H, 6.25\%. Found: C, 82.60; H, 6.47\%.

\{2-Methyl-5-[(E)-2-phenylvinyl]-4,5-dihydrofuran-3-yl\}(phenyl)methanone (3g). Yellow oil, yield 39\%, $113 \mathrm{mg}$, IR (ATR, $\left.\mathrm{cm}^{-1}\right)$ : 3059, $1717(\mathrm{C}=\mathrm{O}), 1700(\mathrm{C}=\mathrm{C}), 1600(\mathrm{C}=\mathrm{C}), 1597,1219$, 749, 692. ${ }^{1} \mathrm{H}$ NMR $\left(300 \mathrm{MHz}, \mathrm{CDCl}_{3}\right), \delta_{\mathrm{H}} 1.94\left(3 \mathrm{H}, \mathrm{t},{ }^{5} J 1.5 \mathrm{~Hz}, \mathrm{CH}_{3}\right), 3.02(1 \mathrm{H}, \mathrm{ddq}, J 14.7,8.4$ and $\left.{ }^{5} J 1.5 \mathrm{~Hz}, \mathrm{H} 4\right), 3.34\left(1 \mathrm{H}\right.$, ddq, $J 14.7,10.2$ and $\left.{ }^{5} J 1.5 \mathrm{~Hz}, \mathrm{H} 4\right), 5.30(1 \mathrm{H}$, ddd, $J 10.0,8.4$ and $7.8 \mathrm{~Hz}, \mathrm{H} 5), 6.34\left(1 \mathrm{H}, \mathrm{dd}, J 15.6\right.$ and $\left.7.2 \mathrm{~Hz}, \mathrm{H}_{\text {olef. }}\right), 6.68\left(1 \mathrm{H}, \mathrm{d}, J 15.6 \mathrm{~Hz}, \mathrm{H}_{\text {olef. }}\right), 7.28-7.36$ $(3 \mathrm{H}, \mathrm{m}), 7.41-7.50(5 \mathrm{H}, \mathrm{m}), 7.60-7.63(2 \mathrm{H}, \mathrm{m}) .{ }^{13} \mathrm{C} \mathrm{NMR}\left(75 \mathrm{MHz}, \mathrm{CDCl}_{3}\right), \delta_{\mathrm{C}} 15.8\left(\mathrm{CH}_{3}\right), 37.6$ (C4), 83.2 (C5), $112.7(\mathrm{C} 3), 127.0,127.7,128.0(2 \mathrm{xCH}), 128.4(2 \mathrm{xCH}), 128.5(2 \times \mathrm{CH}), 128.9$ $(2 \times C H), 131.3,130.0,136.2,141.1,168.9(\mathrm{C} 2), 193.4(\mathrm{C}=\mathrm{O}) . m / z\left(\mathrm{ESI}^{+}\right)=291\left(\mathrm{MH}^{+}, 100 \%\right)$. HRMS $\left(\mathrm{ESI}^{+}\right): \mathrm{m} / z(\mathrm{M}+\mathrm{H})^{+} \quad \mathrm{C}_{20} \mathrm{H}_{19} \mathrm{O}_{2}: 291.13796$ found: 291.14061. Anal. Calcd for $\mathrm{C}_{20} \mathrm{H}_{18} \mathrm{O}_{2}$ (290.35): C, 82.73; H, 6.25\%. Found: C, 82.87; H, 6.15\%.

2-(2,2-Diphenylvinyl)-6,6-dimethyl-3,5,6,7-tetrahydro-1-benzofuran-4(2H)-one $(3 \mathrm{~h}) .{ }^{14}$ Pale yellow solid, yield 91\%, $313 \mathrm{mg}, \mathrm{mp} 113-115^{\circ} \mathrm{C}$, IR (ATR, $\left.\mathrm{cm}^{-1}\right): 2916,1690(\mathrm{C}=\mathrm{O}), 1686$ $(\mathrm{C}=\mathrm{C}), 1073,742,693 .{ }^{1} \mathrm{H}$ NMR $\left(400 \mathrm{MHz}, \mathrm{CDCl}_{3}\right), \delta_{\mathrm{H}} 1.08\left(3 \mathrm{H}, \mathrm{s}, \mathrm{CH}_{3}\right), 1.12\left(3 \mathrm{H}, \mathrm{s}, \mathrm{CH}_{3}\right)$, $2.23(2 \mathrm{H}, \mathrm{s}, \mathrm{H} 5), 2.95(2 \mathrm{H}, \mathrm{t}, J 2.0 \mathrm{~Hz}, \mathrm{H} 7), 2.73(1 \mathrm{H}, \mathrm{dd}, J 14.4$ and $7.6 \mathrm{~Hz}, \mathrm{H} 3), 3.01(1 \mathrm{H}, \mathrm{dd}, J$ 14.4 and $10.4 \mathrm{~Hz}, \mathrm{H} 3), 5.27(1 \mathrm{H}, \mathrm{td}, J 10.0$ and $7.6 \mathrm{~Hz}, \mathrm{H} 2), 6.14(1 \mathrm{H}, \mathrm{d}, J 9.2 \mathrm{~Hz}), 7.21(2 \mathrm{H}$, dd, $J 8.4$ and $2.0 \mathrm{~Hz}), 7.23-7.30(5 \mathrm{H}, \mathrm{m}), 7.36-7.41(3 \mathrm{H}, \mathrm{m}) .{ }^{13} \mathrm{C} \mathrm{NMR}\left(100 \mathrm{MHz}, \mathrm{CDCl}_{3}\right), \delta_{\mathrm{C}} 29.0$ $\left(2 \mathrm{xCH}_{3}\right), 33.2,34.3,38.2,51.2(\mathrm{C} 3), 83.8(\mathrm{C} 2), 111.8(\mathrm{C} 3 \mathrm{a}), 126.5,128.0(2 \mathrm{xCH}), 128.2$ (2xCH), $128.4(2 \times C H), 128.5(2 \times C H), 128.6,130.1,138.8,141.3,146.3,176.4(\mathrm{C} 7 \mathrm{a}), 195.0$ $(\mathrm{C}=\mathrm{O}) . m / z\left(\mathrm{ESI}^{+}\right) 345\left(\mathrm{MH}^{+}, \% 100\right)$. HRMS $\left(\mathrm{ESI}^{+}\right): m / z(\mathrm{M}+\mathrm{H})^{+} \mathrm{C}_{24} \mathrm{H}_{24} \mathrm{O}_{2}: 345.18491$ found: 345.18699 .

1-[5-(2,2-Diphenylvinyl)-2-methyl-4,5-dihydrofuran-3-yl]ethanone (3i). Yellow oil, yield 84\%, $255 \mathrm{mg}$, IR (ATR, $\left.\mathrm{cm}^{-1}\right):$ 3048, 2944, $1628(\mathrm{C}=\mathrm{O}), 1602(\mathrm{C}=\mathrm{C}), 1249,1180,968,728$, 692. ${ }^{1} \mathrm{H}$ NMR $\left(400 \mathrm{MHz}, \mathrm{CDCl}_{3}\right), \delta_{\mathrm{H}} 2.19\left(3 \mathrm{H}, \mathrm{s}, \mathrm{CH}_{3}\right), 2.22\left(3 \mathrm{H}, \mathrm{s}, \mathrm{CH}_{3}\right), 2.86(1 \mathrm{H}, \mathrm{ddq}, J 14.0$, 8.0 and $1.6 \mathrm{~Hz}, \mathrm{H} 4), 3.09(1 \mathrm{H}, \mathrm{ddq}, J 14.4,10.0$ and $1.6 \mathrm{~Hz}, \mathrm{H} 4), 5.10(1 \mathrm{H}, \mathrm{ddd}, J 10.4,9.2$ and $8.0 \mathrm{~Hz}, \mathrm{H} 5), 6.16(1 \mathrm{H}, \mathrm{d}, J 9.2 \mathrm{~Hz}), 7.20(2 \mathrm{H}, \mathrm{dd}, J 8.4$ and $2.0 \mathrm{~Hz}), 7.25-7.29(5 \mathrm{H}, \mathrm{m}), 7.35-7.40$ $(3 \mathrm{H}, \mathrm{m}) .{ }^{13} \mathrm{C}$ NMR $\left(100 \mathrm{MHz}, \mathrm{CDCl}_{3}\right), \delta_{\mathrm{C}} 15.4\left(\mathrm{CH}_{3}\right), 29.7\left(\mathrm{CH}_{3}\right), 37.8(\mathrm{C} 4), 80.3(\mathrm{C} 5), 112.4$ (C3), 126.9, $128.0(2 \mathrm{xCH}), 128.1(2 \mathrm{xCH}), 128.3(2 \mathrm{xCH}), 128.5(2 \times \mathrm{CH}), 128.6,130.1,138.9$, 141.4, 145.8, $167.8(\mathrm{C} 2), 194.6(\mathrm{C}=\mathrm{O}) . \mathrm{m} / z\left(\mathrm{ESI}^{+}\right) 305\left(\mathrm{MH}^{+}, \% 100\right)$. HRMS $\left(\mathrm{ESI}^{+}\right): \mathrm{m} / z$ $(\mathrm{M}+\mathrm{H})^{+} \mathrm{C}_{21} \mathrm{H}_{20} \mathrm{O}_{2}: 305.15361$ found: 305.15616. Anal. Calcd for $\mathrm{C}_{21} \mathrm{H}_{20} \mathrm{O}_{2}$ (304.38): C, 82.86; $\mathrm{H}$, 6.62\%. Found: C, 83. 14; H, 6.55\%.

2,6,6-Trimethyl-2-[(E)-2-phenylvinyl]-3,5,6,7-tetrahydro-1-benzofuran-4(2H)-one (3j). ${ }^{44,45}$ Colourless oil, yield 92\%, $259 \mathrm{mg},{ }^{1} \mathrm{H}$ NMR $\left(300 \mathrm{MHz}, \mathrm{CDCl}_{3}\right), \delta_{\mathrm{H}} 1.07\left(3 \mathrm{H}, \mathrm{s}, \mathrm{CH}_{3}\right), 1.08(3 \mathrm{H}$, s, $\left.\mathrm{CH}_{3}\right), 1.57\left(3 \mathrm{H}, \mathrm{s}, \mathrm{CH}_{3}\right), 2.21(2 \mathrm{H}, \mathrm{s}, \mathrm{H} 5), 2.29(2 \mathrm{H}, \mathrm{s}, \mathrm{H} 7), 2.72(1 \mathrm{H}, \mathrm{d}, J 14.4 \mathrm{~Hz}, \mathrm{H} 3), 2.90$ $(1 \mathrm{H}, \mathrm{d}, J 14.4 \mathrm{~Hz}, \mathrm{H3}), 6.27\left(1 \mathrm{H}, \mathrm{d}, J 15.9 \mathrm{~Hz}, \mathrm{H}_{\text {olef. }}\right), 6.53\left(1 \mathrm{H}, \mathrm{d}, J 15.9 \mathrm{~Hz}, \mathrm{H}_{\text {olef. }}\right), 7.20(1 \mathrm{H}, \mathrm{t}$, $J 6.9 \mathrm{~Hz}), 7.28(2 \mathrm{H}, \mathrm{d}, J 7.5 \mathrm{~Hz}), 7.35(2 \mathrm{H}, \mathrm{d}, J 6.9 \mathrm{~Hz})$. HRMS $\left(\mathrm{ESI}^{+}\right): m / z(\mathrm{M}+\mathrm{H})^{+} \mathrm{C}_{19} \mathrm{H}_{22} \mathrm{O}_{2}$ : 283.16926 found: 283.17136 . 
2-Methyl-2-[(E)-2-phenylvinyl]-3,5,6,7-tetrahydro-1-benzofuran-4(2H)-one (3k). Oil, yield 88\%, $224 \mathrm{mg}$, IR (ATR, $\left.\mathrm{cm}^{-1}\right):$ 3026, 2943, 2866, $1620(\mathrm{C}=\mathrm{O}), 1595(\mathrm{C}=\mathrm{C}), 1244,998,749$, 692. ${ }^{1} \mathrm{H}$ NMR $\left(300 \mathrm{MHz}, \mathrm{CDCl}_{3}\right), \delta_{\mathrm{H}} 1.58\left(3 \mathrm{H}, \mathrm{s}, \mathrm{CH}_{3}\right), 2.02(2 \mathrm{H}, \mathrm{f}, J 6.6 \mathrm{~Hz}, \mathrm{H} 6), 2.34(1 \mathrm{H}, \mathrm{t}, J$ $6.9 \mathrm{~Hz}, \mathrm{H} 5), 2.44(2 \mathrm{H}, \mathrm{td}, J 6.9$ and $1.5 \mathrm{~Hz}, \mathrm{H} 7), 2.71(1 \mathrm{H}, \mathrm{td}, J 14.4$ and $1.8 \mathrm{~Hz}, \mathrm{H} 3), 2.90(1 \mathrm{H}$, td, $J 14.4$ and $1.8 \mathrm{~Hz}, \mathrm{H} 3), 6.29\left(1 \mathrm{H}, \mathrm{d}, J 15.9 \mathrm{~Hz}, \mathrm{H}_{\text {olef. }}\right), 6.55\left(1 \mathrm{H}, \mathrm{d}, J 15.9 \mathrm{~Hz}, \mathrm{H}_{\text {olef. }}\right), 7.24$ $(1 \mathrm{H}, \mathrm{tt}, J 6.6$ and $1.5 \mathrm{~Hz}), 7.29(2 \mathrm{H}, \mathrm{t}, J 6.9 \mathrm{~Hz}), 7.35(2 \mathrm{H}, \mathrm{dd}, J 6.9$ and $1.8 \mathrm{~Hz}) .{ }^{13} \mathrm{C}$ NMR $(75$ $\left.\mathrm{MHz}, \mathrm{CDCl}_{3}\right), \delta_{\mathrm{C}} 21.9\left(\mathrm{CH}_{3}\right), 24.4,27.1,36.6,38.6(\mathrm{C} 3), 91.5(\mathrm{C} 3 \mathrm{a}), 112.4(\mathrm{C} 2), 126.9,128.2$ $(2 \mathrm{xCH}), 128.7(2 \mathrm{xCH}), 128.8,132.1,136.3,176.4(\mathrm{C} 7 \mathrm{a}), 196.0(\mathrm{C}=\mathrm{O}) . \mathrm{m} / z\left(\mathrm{ESI}^{+}\right) 255\left(\mathrm{MH}^{+}\right.$, \%100). HRMS (ESI $\left.{ }^{+}\right): m / z(\mathrm{M}+\mathrm{H})^{+} \mathrm{C}_{17} \mathrm{H}_{18} \mathrm{O}_{2}: 255.13795$ found: 255.13822. Anal. Calcd for $\mathrm{C}_{17} \mathrm{H}_{18} \mathrm{O}_{2}$ (254.32): C, 80.28; H, 7.13\%. Found: C, 80.36; H, 7.43\%.

2-Methyl-6-phenyl-2-[(E)-2-phenylvinyl]-3,5,6,7-tetrahydro-1-benzofuran-4(2H)-one (3I). Colourless solid, yield 82\%, $271 \mathrm{mg}, \mathrm{mp} \mathrm{158-160}{ }^{\circ} \mathrm{C}$, IR (ATR, $\mathrm{cm}^{-1}$ ): 2977, 1649 (C=O), 1623 $(\mathrm{C}=\mathrm{C}), 1218,1028,750,690 .{ }^{1} \mathrm{H}$ NMR $\left(300 \mathrm{MHz}, \mathrm{CDCl}_{3}\right), \delta_{\mathrm{H}} 1.65\left(3 \mathrm{H}, \mathrm{s}, \mathrm{CH}_{3}\right), 2.65(2 \mathrm{H}, \mathrm{dd}, J$ 9.0 and $2.4 \mathrm{~Hz}, \mathrm{H} 5), 2.68-2.73(2 \mathrm{H}, \mathrm{m}, \mathrm{H} 7), 2.80$ (1H, ddd, $J 14.4,4.2$ and $1.5 \mathrm{~Hz}, \mathrm{H} 3), 2.98$ $(1 \mathrm{H}, \mathrm{ddd}, J 14.4,4.2$ and $1.5 \mathrm{~Hz}, \mathrm{H} 3), 3.46(1 \mathrm{H}, \mathrm{m}, \mathrm{H} 6), 6.32\left(1 \mathrm{H}, \mathrm{d}, J 15.9 \mathrm{~Hz}, \mathrm{H}_{\mathrm{olef}}\right), 6.55(1 \mathrm{H}$, d, J 15.9 Hz, H olef. $_{1}, 7.25-7.40(10 \mathrm{H}, \mathrm{m}) .{ }^{13} \mathrm{C} \mathrm{NMR}\left(75 \mathrm{MHz}, \mathrm{CDCl}_{3}\right), \delta_{\mathrm{C}} 27.1\left(\mathrm{CH}_{3}\right), 31.9,38.5$, 40.6, 44.0 (C2), 92.2 (C3a), 112.6, 126.9, $127.0(2 \mathrm{xCH}), 127.3(2 \times C H), 128.3(2 \times C H)$, 128.9(2xCH), 129.0, 132.0, 136.2, 142.9, $175.5(\mathrm{C} 7 \mathrm{a}), 194.5(\mathrm{C}=\mathrm{O}) . m / z\left(\mathrm{ESI}^{+}\right) 331\left(\mathrm{MH}^{+}\right.$, \%100). HRMS (ESI $\left.{ }^{+}\right): m / z(M+H)^{+} \mathrm{C}_{23} \mathrm{H}_{22} \mathrm{O}_{2}: 331.16926$ found: 331.16895. Anal. Calcd for $\mathrm{C}_{23} \mathrm{H}_{22} \mathrm{O}_{2}$ (330.41): C, 83.60; H, 6.71\%. Found: C, 83.91; H, 6.88\%.

1-\{2,5-Dimethyl-5-[(E)-2-phenylvinyl]-4,5-dihydrofuran-3-yl\}ethanone (3m). ${ }^{45}$ Yellow oil, yield 75\%, $181 \mathrm{mg},{ }^{1} \mathrm{H}$ NMR $\left(300 \mathrm{MHz}, \mathrm{CDCl}_{3}\right), \delta_{\mathrm{H}} 1.57\left(3 \mathrm{H}, \mathrm{s}, \mathrm{CH}_{3}\right), 2.27(3 \mathrm{H}, \mathrm{t}, J 1.5 \mathrm{~Hz}$, $\left.\mathrm{CH}_{3}\right), 2.86(1 \mathrm{H}, \mathrm{dq}, J 14.1$ and $1.5 \mathrm{~Hz}, \mathrm{H} 3), 3.02(1 \mathrm{H}, \mathrm{dq}, J 14.1$ and $1.5 \mathrm{~Hz}, \mathrm{H} 3), 6.30(1 \mathrm{H}, \mathrm{d}, J$ $\left.16.2 \mathrm{~Hz}, \mathrm{H}_{\text {olef. }}\right), 6.55\left(1 \mathrm{H}, \mathrm{d}, J 16.2 \mathrm{~Hz}, \mathrm{H}_{\text {olef. }}\right), 7.27(1 \mathrm{H}, \mathrm{tt}, J 6.9$ and $1.5 \mathrm{~Hz}), 7.31(2 \mathrm{H}, \mathrm{t}, J 6.9$ $\mathrm{Hz}), 7.38(2 \mathrm{H}$, dd, $J 6.9$ and $1.8 \mathrm{~Hz})$. HRMS $\left(\mathrm{ESI}^{+}\right): m / z(\mathrm{M}+\mathrm{H})^{+} \mathrm{C}_{16} \mathrm{H}_{18} \mathrm{O}_{2}: 243.13796$ found: 243.13702 .

Ethyl 2,5-dimethyl-5-[(E)-2-phenylvinyl]-4,5-dihydrofuran-3-carboxylate (3n). ${ }^{45}$ Pale yellow oil, yield 71\%, $193 \mathrm{mg},{ }^{1} \mathrm{H}$ NMR $\left(300 \mathrm{MHz}, \mathrm{CDCl}_{3}\right), \delta_{\mathrm{H}} 1.27\left(3 \mathrm{H}, \mathrm{t}, J 7.2 \mathrm{~Hz},-\mathrm{OCH}_{2} \mathrm{CH}_{3}\right), 1.55$ $\left(3 \mathrm{H}, \mathrm{s}, \mathrm{CH}_{3}\right), 2.24\left(3 \mathrm{H}, \mathrm{t}, J 1.5 \mathrm{~Hz}, \mathrm{CH}_{3}\right), 2.80(1 \mathrm{H}, \mathrm{dq}, J 14.4$ and $1.5 \mathrm{~Hz}, \mathrm{H} 3), 2.97(1 \mathrm{H}, \mathrm{dq}, J$ 14.4 and $1.5 \mathrm{~Hz}, \mathrm{H} 3), 4.16\left(2 \mathrm{H}, \mathrm{q}, J 7.2 \mathrm{~Hz},-\mathrm{OCH}_{2} \mathrm{CH}_{3}\right), 6.29\left(1 \mathrm{H}, \mathrm{d}, J 16.2 \mathrm{~Hz}, \mathrm{H}_{\text {olef. }}\right), 6.54$ $\left(1 \mathrm{H}, \mathrm{d}, J 16.2 \mathrm{~Hz}, \mathrm{H}_{\mathrm{olef}}\right), 7.24(1 \mathrm{H}, \mathrm{tt}, J 7.2$ and $1.2 \mathrm{~Hz}), 7.30(2 \mathrm{H}, \mathrm{t}, J 6.9 \mathrm{~Hz}), 7.37(2 \mathrm{H}, \mathrm{dd}, J$ 6.9 and $1.2 \mathrm{~Hz})$. HRMS (ESI $\left.{ }^{+}\right): m / z(\mathrm{M}+\mathrm{H})^{+} \mathrm{C}_{17} \mathrm{H}_{20} \mathrm{O}_{3}: 273.14852$ found: 273.14841.

1-\{5-Methyl-2-phenyl-5-[(E)-2-phenylvinyl]-4,5-dihydrofuran-3-yl\}ethanone (3o). Yellow oil, yield 40\%, $122 \mathrm{mg}$, IR (ATR, $\left.\mathrm{cm}^{-1}\right)$ : 3018, $1716(\mathrm{C}=\mathrm{O}), 1600(\mathrm{C}=\mathrm{C}), 1587(\mathrm{C}=\mathrm{C}), 1241$, 749, 693. ${ }^{1} \mathrm{H}$ NMR $\left(300 \mathrm{MHz}, \mathrm{CDCl}_{3}\right), \delta_{\mathrm{H}} 1.59\left(3 \mathrm{H}, \mathrm{s}, \mathrm{CH}_{3}\right), 1.88\left(3 \mathrm{H}, \mathrm{s}, \mathrm{CH}_{3}\right), 2.96(1 \mathrm{H}, \mathrm{d}, J$ $14.4 \mathrm{~Hz}, \mathrm{H} 3), 3.20$ (1H, d, J 14.4 Hz, H3), 6.30 (1H, d, J $\left.16.2 \mathrm{~Hz}, \mathrm{H}_{\text {olef. }}\right), 6.53$ (1H, d, J 16.2 Hz, $\left.\mathrm{H}_{\text {olef. }}\right), 7.25(2 \mathrm{H}, \mathrm{t}, J 6.9 \mathrm{~Hz}), 7.31-7.39(5 \mathrm{H}, \mathrm{m}), 7.56(3 \mathrm{H}, \mathrm{m}) .{ }^{13} \mathrm{C} \mathrm{NMR}\left(75 \mathrm{MHz}, \mathrm{CDCl}_{3}\right), \delta_{\mathrm{C}}$ $15.9\left(\mathrm{CH}_{3}\right), 27.0\left(\mathrm{CH}_{3}\right), 43.6(\mathrm{C} 4), 87.6(\mathrm{C} 5), 114.6(\mathrm{C} 3), 126.8,127.9,127.9(2 \times \mathrm{CH}), 128.1$ $(2 \mathrm{xCH}), 128.4(2 \mathrm{xCH}), 128.8(2 \mathrm{xCH}), 129.4,131.1,132.6,136.5,141.2,168.0(\mathrm{C} 2), 195.1$ 
$(\mathrm{C}=\mathrm{O}) . m / z\left(\mathrm{ESI}^{+}\right) 305\left(\mathrm{MH}^{+}, \% 100\right)$. HRMS $\left(\mathrm{ESI}^{+}\right): m / z(\mathrm{M}+\mathrm{H})^{+} \mathrm{C}_{21} \mathrm{H}_{20} \mathrm{O}_{2}: 305.15361$ found: 305.15495. Anal. Calcd for $\mathrm{C}_{21} \mathrm{H}_{20} \mathrm{O}_{2}$ (304.38): C, 82.86; H, 6.62\%. Found: $\mathrm{C}, 83.06 ; \mathrm{H}, 6.84 \%$.

\{2,5-Dimethyl-5-[(E)-2-phenylvinyl]-4,5-dihydrofuran-3-yl\}(phenyl)methanone (3p). Yellow oil, yield 38\%, $116 \mathrm{mg}$, IR (ATR, $\mathrm{cm}^{-1}$ ): 3027, 2974, 2927, 1650 (C=O), 1593 (C=C), 1239, 746, 693. ${ }^{1} \mathrm{H}$ NMR $\left(400 \mathrm{MHz}, \mathrm{CDCl}_{3}\right), \delta_{\mathrm{H}} 1.62\left(3 \mathrm{H}, \mathrm{s}, \mathrm{CH}_{3}\right), 1.89\left(3 \mathrm{H}, \mathrm{t}, J 1.6 \mathrm{~Hz}, \mathrm{CH}_{3}\right), 3.0(1 \mathrm{H}, \mathrm{dq}$, $J 14.4$ and $1.6 \mathrm{~Hz}, \mathrm{H} 3), 3.20(1 \mathrm{H}, \mathrm{dq}, J 14.4$ and $1.6 \mathrm{~Hz}, \mathrm{H} 3), 6.35\left(1 \mathrm{H}, \mathrm{d}, J 16.0 \mathrm{~Hz}, \mathrm{H}_{\text {olef. }}\right), 6.60$ $\left(1 \mathrm{H}, \mathrm{d}, J 16.0 \mathrm{~Hz}, \mathrm{H}_{\text {olef. }}\right), 7.32(2 \mathrm{H}, \mathrm{t}, J 7.6 \mathrm{~Hz}), 7.39-7.42(5 \mathrm{H}, \mathrm{m}), 7.56(3 \mathrm{H}, \mathrm{m}) .{ }^{13} \mathrm{C}$ NMR $(100$ $\left.\mathrm{MHz}, \mathrm{CDCl}_{3}\right), \delta_{\mathrm{C}} 15.7\left(\mathrm{CH}_{3}\right), 27.1\left(\mathrm{CH}_{3}\right), 43.4(\mathrm{C} 4), 87.4(\mathrm{C} 5), 112.0(\mathrm{C} 3), 126.6,127.7,127.9$ $(2 \times C H), 128.1(2 \times C H), 128.3(2 \times C H), 128.6(2 \times C H), 132.1,132.4,136.2,141.0,167.7(\mathrm{C} 2)$, $193.2(\mathrm{C}=\mathrm{O}) . \mathrm{m} / z\left(\mathrm{ESI}^{+}\right) 305\left(\mathrm{MH}^{+}, \% 100\right)$. HRMS $\left(\mathrm{ESI}^{+}\right): \mathrm{m} / z(\mathrm{M}+\mathrm{H})^{+} \mathrm{C}_{21} \mathrm{H}_{20} \mathrm{O}_{2}: 305.15361$ found: 305.15534. Anal. Calcd for $\mathrm{C}_{21} \mathrm{H}_{20} \mathrm{O}_{2}$ (304.38): C, 82.86; H, 6.62\%. Found: C, 83.12; H, $6.41 \%$.

\section{Acknowledgements}

The authors are grateful to the Kocaeli University (BAP 2014/18) Science Research Foundations for financial support. Also, authors thank to UNAM (Bilkent University) for HRMS spectra. A. Ustalar thanks to TUBITAK for doctoral fellowship.

\section{References}

1. Garzino, F.; Méou, A.; Brun, P. Tetrahedron Lett. 2000, 41, 9803-9807. http://dx.doi.org/10.1016/S0040-4039(00)01729-9

2. Gregory, B.; Parsons, A. F.; Thomas, C. B. Tetrahedron Lett. 2000, 41, 7751-7755. http://dx.doi.org/10.1016/S0040-4039(00)01729-9

3. Kajikawa, S.; Nishino, H.; Kurosawa, K. Heterocycles 2001, 54, 171-183. http://dx.doi.org/10.3987/COM-00-S(I) 14

4. Gregory, B.; Parsons, A. F.; Thomas, C. B. Tetrahedron 2001, 57, 4719-4728. http://dx.doi.org/10.1016/S0040-4020(01)00375-1

5. Melikyan, G. G.Synthesis 1993, 833-850. http://dx.doi.org/10.1055/s-1993-25951

6. Igbal, J.; Bhatia, B.; Nayyar, N. K. Chem. Rev. 1994, 94, 519-564. http://dx.doi.org/10.1021/cr00026a008

7. Snider, B. B. Chem. Rev. 1996, 96, 339-363. http://dx.doi.org/10.1021/cr950026m PMid:11848756

8. Wang, G.-W.; Dong, Y.-W.; Wu, P.; Yuan, T.-T.; Shen, Y.-B. J. Org. Chem. 2008, 73, 70887095.

\section{http://dx.doi.org/10.1021/jo800870z}


9. Demir, A. S.; Emrullahoglu, M. Curr. Org. Synth. 2007, 4, 223-237. http://dx.doi.org/10.2174/157017907780598871

10. Caliskan, R.; Pekel, T.; Watson, W. H.; Balci, M. Tetrahedron Lett. 2005, 46, 6727-6230. http://dx.doi.org/10.1016/j.tetlet.2005.07.051

11. Chuang, C-P.; Tsai, A-I. Tetrahedron 2013, 69, 3293-3301. http://dx.doi.org/10.1016/j.tet.2013.02.009

12. Kim, S. H.; Kim, S. H.; Lim, C. H.; Kim, J. N. Tetrahedron Lett. 2013, 54, 1697-1699. http://dx.doi.org/10.1016/j.tetlet.2013.01.056

13. Aslan, H.; Akpinar, D. E.; Oktemer, A.; Yakut, M.; Alagoz, O. Helv. Chim. Acta 2014, 97 , $652-661$. http://dx.doi.org/10.1002/hlca.201300229

14. Nishino, H.; Kumabe, R.; Hamada, R.; Yakut, M. Tetrahedron 2014, 70, 1437-50. http://dx.doi.org/10.1016/j.tet.2014.01.013

15. Lee, Y. R.; Kim, B. S.; Kim, D. H. Tetrahedron 2000, 56, 8845-8853. http://dx.doi.org/10.1016/S0040-4020(00)00839-5

16. Nair, V.; Treesa, P. M.; Maliakal, D.; Rath, N. P. Tetrahedron 2001, 57, 7705-7710. http://dx.doi.org/10.1016/S0040-4020(01)00700-1

17. Tseng, C.-H.; Wu, Y.-L.; Chuang, C.-P. Tetrahedron 2002, 58, 7625-7633. http://dx.doi.org/10.1016/S0040-4020(02)00864-5

18. Liao, Y.-J.; Wu, Y.-L.; Chuang, C.-P. Tetrahedron 2003, 59, 3511-3520. http://dx.doi.org/10.1016/S0040-4020(03)00486-1

19. Kobayashi, K.; Nagase, K.; Morikawa, O.; Konishi, H. Heterocycles 2003, 60, 939-946. http://dx.doi.org/10.3987/COM-02-9690

20. Wu, Y.-L.; Chuang, C.-P. Tetrahedron 2004, 60, 1841-1847. http://dx.doi.org/10.1016/j.tet.2003.12.035

21. Nair, V.; Deepthi, A. Chem. Rev. C 2007, 107, 1862-1891. http://dx.doi.org/10.1021/cr068408n

22. Nair, V.; Mohanan, K.; Suja, T. D.; Suresh, E. Tetrahedron Lett. 2006, 47, 2803-2806. http://dx.doi.org/10.1016/j.tetlet.2006.02.051

23. Kajikawa, S.; Nishino, H.; Kurosawa, K. Heterocycles 2001, 54, 171-183. http://dx.doi.org/10.3987/COM-00-S(I)14

24. Bahrami, K.; Khodaei, M. M.; Naali, F. J. Org. Chem. 2008, 73, 6835-6837. http://dx.doi.org/10.1021/jo8010232

25. Yilmaz, M.; Yakut, M.; Pekel, A. T. Synth. Commun. 2008, 38, 914-927. http://dx.doi.org/10.1080/00397910701845456

26. Alagoz, O.; Yilmaz, M.; Pekel, A.T.; Graiff, C.; Maggi, R. RSC Adv. 2014, 414644. http://dx.doi.org/10.1039/C3RA48015A

27. Yilmaz, M.; Yilmaz, E. V. B.; Pekel, A. T. Helv. Chim. Acta 2011, 94, 2027-2038. http://dx.doi.org/10.1002/hlca.201100105

28. Yilmaz, M. Tetrahedron 2011, 67, 8255-8263. 
http://dx.doi.org/10.1016/j.tet.2011.08.098

29. Yilmaz, M.; Pekel, A. T. J. Fluorine Chem. 2005, 126, 401-406. http://dx.doi.org/10.1016/j.jfluchem.2005.02.002

30. Yilmaz, M.; Pekel, A. T. J. Fluorine Chem. 2011, 132, 628-635. http://dx.doi.org/10.1016/j.jfluchem.2011.06.023

31. Bicer, E.; Yilmaz, M. Arkivoc 2013, (iii), 304-316. http://www.arkat-usa.org/get-file/47725/

32. Alagoz, O.; Yilmaz, M.; Pekel, A. T. Synth. Commun. 2006, 36, 1005-1013. http://dx.doi.org/10.1080/00397910500501516

33. Yilmaz, M.; Bicer, E.; Pekel, A. T. Turk. J. Chem. 2005, 29, 579-587. http://journals.tubitak.gov.tr/chem/issues/kim-08-32-4/kim-32-4-2-0708-25.pdf

34. Yilmaz, M.; Bicer, E.; Ustalar, A.; Pekel, A.T. Arkivoc 2014 (v) 225-236. http://dx.doi.org/10.3998/ark.5550190.0015.500

35. Bicer, E.; Yilmaz, M; Karatas, M.; Pekel, A. T. Helv. Chim. Acta 2012, 95, 795-804. http://dx.doi.org/10.1002/hlca.201100397

36. Bicer, E.; Yilmaz, M.; Burgaz, E. V.; Pekel, A. T. Helv. Chim. Acta 2013, 96, 135-141. http://dx.doi.org/10.1002/hlca.201200098

37. Yilmaz, M.; Uzunalioglu, N.; Pekel, A. T. Tetrahedron 2005, 61, 8860-8867. http://dx.doi.org/10.1016/j.tet.2005.07.019

38. Yilmaz, M.; Uzunalioglu, N.; Yakut, M.; Pekel, A. T. Turk. J. Chem. 2008, 32, 411-422. http://journals.tubitak.gov.tr/chem/issues/kim-08-32-4/kim-32-4-2-0708-25.pdf

39. Yilmaz, M.; Burgaz, E. V.; Yakut, M.; Bicer, E. J. Chin. Chem. Soc. 2014, 61, 1101-1107. http://dx.doi.org/10.1002/jccs.201400173

40. Burgaz, E. V.; Yilmaz, M.; Pekel, A. T.; Oktemer, A. Tetrahedron 2007, 63, 7229-7239. http://dx.doi.org/10.1016/j.tet.2007.04.088

41. Yilmaz, E.V.B.; Yilmaz, M.; Oktemer, A. Arkivoc 2011, (ii), 363-376. http://dx.doi.org/10.3998/ark.5550190.0012.230

42. Yilmaz, M. Helv. Chim. Acta 2011, 94, 1335-1342. http://dx.doi.org/10.1002/hlca.201000440

43. Sellanes, D.; Scarone, L.; Manta, E.; Wipf, P.; Sera, G. Lett. Org. Chem. 2006, 3, 309-312. http://dx.doi.org/10.2174/157017806776114621

44. Lebel, H.; Guayt, D.; Paquet, U.; Huard, K. Org. Lett. 2004, 6, 3047-3050. http://dx.doi.org/10.1021/o1049085p

45. Lambert, J. B.; Liu, C.; Kouliev, T. J. Phys. Org. Chem. 2002, 15, 667-671. http://dx.doi.org/10.1002/poc.531

46. Karade, N. N.; Shirodkar, S. G.; Patil, M. N.; Potrekarb, R. A.; Karadeb, H. N. Tetrahedron Lett. 2003, 44, 6729-6731. http://dx.doi.org/10.1016/S0040-4039(03)01644-7

47. Nair, V.; Nair, L. G.; Balagopal, L.; Mathew, J. Ind. J. Chem. Sec. B, 2000, 39B, 352-356. 
http://nopr.niscair.res.in/bitstream/123456789/22523/1/IJCB\%2039B\%285\%29\%20352356.pdf

48. Jiao, J.; Zhang, Y.; Devery, J. J.; Xu, L.; Deng, J,; Flowers , R. A. J. Org. Chem. 2007, 72, 5486-5492.

http://dx.doi.org/10.1021/jo0625406 